\title{
Local regular dual reciprocity method for 2D convection-diffusion equation
}

\author{
N. Caruso ${ }^{1,2}$, M. Portapila ${ }^{1,2} \&$ H. Power ${ }^{3}$ \\ ${ }^{1}$ CURIHAM, FCEIA, University of Rosario, Argentina \\ ${ }^{2}$ CIFASIS, CONICET, UNR-AMU, Argentina \\ ${ }^{3}$ Department of Mechanical Engineering, University of Nottingham, UK
}

\begin{abstract}
In this paper a new technique considering the dual reciprocity method (DRM) with only internal collocation points is considered along with local radial basis function interpolation. This approach gives rise to regular integral equations. Numerical results for the convection-diffusion equation are presented for different Peclet numbers. Comparisons with other numerical techniques are shown in order to illustrate the good solutions obtained by this method.
\end{abstract}

Keywords: DRM, RBF, regular integral equations.

\section{Introduction}

The basis of boundary element method (BEM) is to transform the original partial differential equation (PDE), into an equivalent integral equation. Several methods have been developed to take domain integrals to the boundary in order to eliminate the need for internal cells (boundary only BEM formulations). One of the most popular to date is the dual reciprocity method (DRM) introduced by Nardini and Brebbia [1], it converts the domain integrals into equivalent boundary integrals.

Popov and Power [2] found that the DRM approach can be substantially improved by using domain decomposition to improve the accuracy of the DRM approach, this idea was inspired by the work of Kansa and Carlson [3] on the radial basis function (RBF) data approximations. Florez et al. [4] improved the performance of the DRM in the BEM numerical solution of the NavierStokes equations through a multidomain decomposition technique. Following those results, the performance the DRM-MD was investigated [5], implementing quadratic shape functions of the boundary elements for both the approximation of 
the geometry and the surface variables of the governing equations. In recent years, the theory of radial basis functions (RBFs) has undergone intensive research and enjoyed considerable success as a technique for interpolating multivariable data and functions. The idea of introducing RBF interpolation to improve the accuracy of a classical numerical scheme has been employed by Wright and Fornberg [6].

In [7] a modified Control Volume (CV) method which uses a RBF interpolation to improve the prediction of the flux accuracy at the faces of the $\mathrm{CV}$ is presented. This method is also more flexible than the classical CV formulations because the boundary conditions are explicitly imposed in the interpolation formula, without the need for artificial schemes (e.g. utilising dummy cells).

Popov and Bui [8] introduced the radial basis integral equation method (RBIEM). It naturally extends from the work of Popov and Bui [9] by adopting circular subdomains and by using interpolation to obtain the distributions over the local boundaries. The RBIEM evaluates the derivatives by employing two in 2D problems additional equations obtained by differentiating the local integral equation. In this work a new DRM technique is introduced where only regular integral equations are considered and local RBF interpolation is implemented.

\section{Mathematical formulation}

Let us consider a boundary problem on the domain $\Omega$. The governing partial differential equation for this problem can be written as:

$$
\nabla^{2} u(\mathbf{x})=b\left(\mathbf{x}, u(\mathbf{x}), \frac{\partial u}{\partial x_{i}}(\mathbf{x})\right)
$$

where $u(\mathbf{x})$ is the potential field, $\mathbf{x}$ the position vector, $x_{i}$ the $\mathrm{i}$ component of $\mathbf{x}$. The problem definition is completed by specifying the following boundary conditions $(\mathrm{BC})$ on $\Gamma(\partial \Omega)$ :

$$
\begin{gathered}
u(\mathbf{x})=u_{0}(\mathbf{x}) \quad \text { on } \Gamma_{1} \\
\frac{\partial u}{\partial n}(\mathbf{x})=q_{0}(\mathbf{x}) \quad \text { on } \Gamma_{2}
\end{gathered}
$$

where $\Gamma_{1} \cup \Gamma_{2}=\Gamma$, and $\Gamma_{1}$ and $\Gamma_{2}$ are non-intersecting parts of the boundary, the functions $u_{0}$ and $q_{0}$ are suitably prescribed functions of $\mathbf{x}$.

Integral equations for linear problems can be formulated through the application of Green's identities, which represent a harmonic function as the superposition of a single-layer, a double-layer potential and a volume potential.

If $b=0$ the governing equation (1) is the Laplace equation. By using the Green's identities, the integral representation over the Laplace equation its integral representation can be written as:

$$
c(\xi) u(\xi)=\int_{\Gamma} q^{*}(\mathbf{x}, \xi) u(\mathbf{x}) d \Gamma_{x}-\int_{\Gamma} u^{*}(\mathbf{x}, \xi) q(\mathbf{x}) d \Gamma_{x}
$$

where $\xi$ is a source point, and $u^{*}(\mathbf{x}, \xi)$ is the fundamental solution of the Laplace problem, $\nabla^{2} u^{*}(\mathbf{x})=\delta(\mathbf{x}, \xi)$ where $\delta(\mathbf{x}, \xi)$ is the Delta function. The Green's 
function (called here the fundamental solution) $\mathrm{u}^{*}$ satisfying this equation, is given by:

$$
u^{*}(\mathbf{x}, \xi)=\frac{1}{2 \pi} \ln \left(\frac{1}{R}\right)
$$

for 2D problem, where $R$ is the distance from the source point, $R=|\mathbf{x}-\xi|$ and $q^{*}(\mathbf{x})=\frac{\partial u^{*}}{\partial n}(\mathbf{x}, \xi)$. The constant value $c(\xi) \in[0,1]$, being 1 if the point $\mathbf{x}$ is inside the domain and $\frac{1}{2}$ if the point $\mathbf{x}$ is on a smooth part of the domain boundary $\Gamma$.

For generals $b\left(\mathbf{x}, u, \frac{\partial u}{\partial n}\right)$ in order to obtain the similar integral eq. (4) the fundamental solution is available only for a few simple forms of $b$, for more complicated function $u^{*}$ is not available.

In this work the following equation is considered:

$$
D \frac{\partial^{2} u(\mathbf{x})}{\partial x_{j} x_{i}}+V_{i} \frac{\partial u(\mathbf{x})}{\partial x_{i}}+k u(\mathbf{x})=0
$$

with no fundamental solution available for arbitrary values of the convective velocity and reaction terms, the following integral formulation is used:

$$
c(\xi) u(\xi)=\int_{\Gamma} q^{*}(\mathbf{x}, \xi) u(\mathbf{x}) d \Gamma_{x}-\int_{\Gamma} u^{*}(\mathbf{x}, \xi) q(\mathbf{x}) d \Gamma_{x}-\int_{\Omega} b u^{*}(\mathbf{x}, \xi) d \Omega_{x}
$$

with $b=-V_{i} \frac{\partial u}{\partial x_{i}}(\mathbf{x})-k u(\mathbf{x})$. Equation (7) consists of both surface and domain integrals.

Several methods have been developed to take domain integrals to the boundary, in this work the Dual Reciprocity Method (DRM) is considered [1], which most remarkable feature is the fact that it does not depend on obtaining a new particular solutions for each case under consideration. The DRM employs an RBF approximation of the non-homogeneous term $b$, such that:

$$
b \approx \sum_{k=1}^{N} \beta_{k} \varphi\left(\mathbf{x}, \mathbf{x}_{\mathbf{k}}\right)
$$

substituting (8) into the domain integral in eq. (7) yields:

$$
\int_{\Omega} b(\mathbf{x}) u^{*}(\mathbf{x}, \xi) d \Omega_{x} \approx \sum_{k=1}^{N} \beta_{k} \varphi\left(\mathbf{x}, \mathbf{x}_{\mathbf{k}}\right) u^{*}(\mathbf{x}, \xi) d \Omega_{x}
$$

if a particular solution $\widetilde{\varphi}\left(\mathbf{x}, \mathbf{x}_{\mathbf{k}}\right)$ exists such that: $\nabla^{2} \widetilde{\varphi}\left(\mathbf{x}, \mathbf{x}_{\mathbf{k}}\right)=\varphi\left(\mathbf{x}, \mathbf{x}_{\mathbf{k}}\right)$. by replacing into (9) and applying Green's second identity in eq. (7) it is finally 
obtained:

$$
\begin{aligned}
c(\xi) u(\xi)= & \int_{\Gamma} q^{*}(\mathbf{x}, \xi) u(\mathbf{x}) d \Gamma_{x}-\int_{\Gamma} u^{*}(\mathbf{x}, \xi) q(\mathbf{x}) d \Gamma_{x} \\
& -\sum_{k=1}^{N} \beta_{k}\left\{c(\xi) \widetilde{\varphi}\left(\xi, \mathbf{x}_{\mathbf{k}}\right)-\int_{\Gamma} q^{*}(\mathbf{x}, \xi) \widetilde{\varphi}\left(\mathbf{x}, \mathbf{x}_{\mathbf{k}}\right) d \Gamma_{x}\right. \\
& \left.+\int_{\Gamma} u^{*}(\mathbf{x}, \xi) \frac{\widetilde{\partial \varphi}}{\partial n}\left(\mathbf{x}, \mathbf{x}_{\mathbf{k}}\right) d \Gamma_{x}\right\}
\end{aligned}
$$

A radial basis function $(\mathrm{RBF}), \varphi_{j}(\mathbf{x})=\varphi\left(\mathbf{x}-\mathbf{x}_{\mathbf{k}}\right)=\varphi\left(\left\|\mathbf{x}-\mathbf{x}_{\mathbf{k}}\right\|\right)$ depends upon the separation distances of a subset of data centres. In a typical interpolation problem $N$ pairs of data points $\left\{\left(x_{j}, F\left(x_{j}\right)\right)_{j=1}^{N}\right\}$, which are assumed to be datas of one unknown function $F$ that is to be interpolated by the function $f$, i.e.

$$
f(\mathbf{x})=\sum_{j=1}^{N} \alpha_{j} \varphi\left(\left\|\mathbf{x}-\mathbf{x}_{\mathbf{j}}\right\|\right)+P_{m-1}(x)
$$

along with the constraint conditions

$$
\sum_{j=1}^{N} \alpha_{j} p_{k}\left(x_{j}\right)=0 \quad 1 \leq k \leq m-1
$$

where $\alpha_{j}, j=1, \ldots, N$ are real coefficients and $\varphi$ is a radial basis function. The matrix formulation of the above interpolation problem can be written as $A x=b$ with

$$
A=\left(\begin{array}{cc}
\Psi & P_{m-1} \\
P_{m-1}^{t} & 0
\end{array}\right)
$$

\subsection{Regular local dual reciprocity method scheme}

In the proposed formulation a decomposition of $\Omega$ in subdomains is considered (Fig. 1). In this work only rectangular sub-domains with variable number of internal points will be considered. Even though other possibilities are possible, such as overlapping sub-domains and any geometrical shape per sub-domain. Which gives the possibility of extending this method to more complex and general conditions.

In the proposed scheme the integral eq. (7) is applied to one or more than one source points inside the sub-domains. As no source points are considered on the boundaries of the local sub-domains the obtained integral equation will always be regular. 


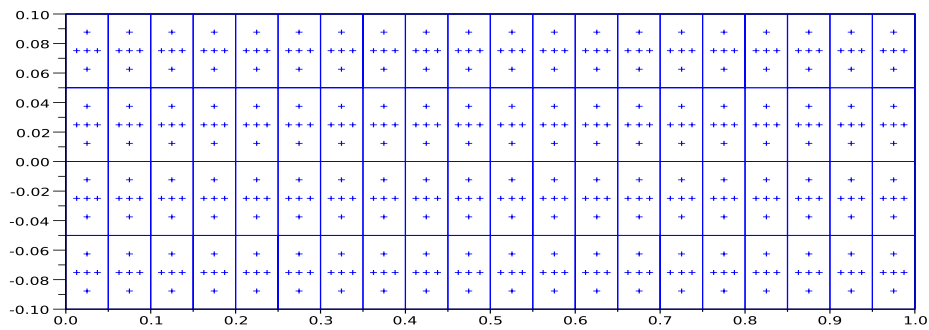

Figure 1: uniform discretization of the domain: 80 sub-domains, 5 internal points per sub-domain.

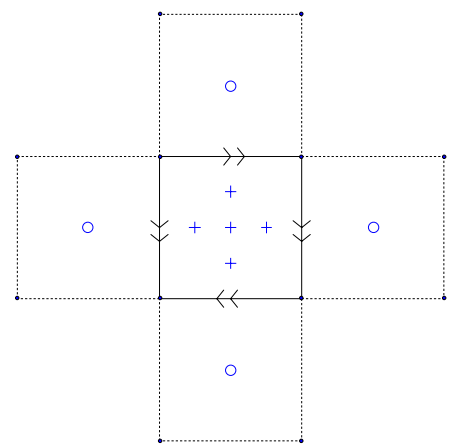

Stencil A

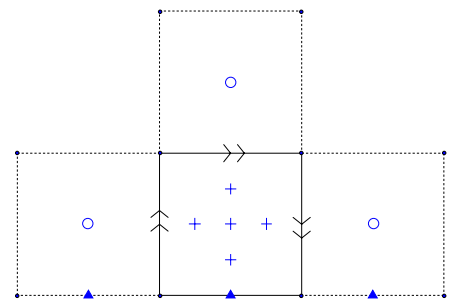

Stencil C

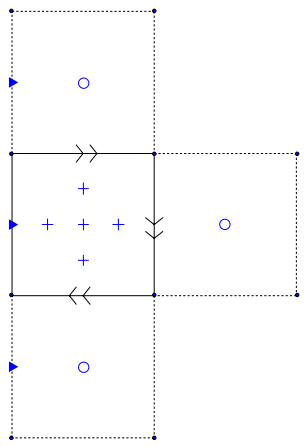

Stencil B

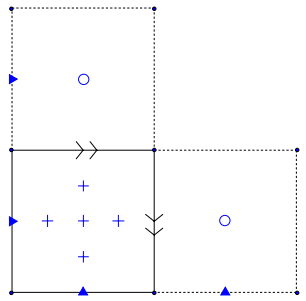

Stencil D

Figure 2: Stencils and set of points configuration. Crosses: collocation and interpolation points. Circles: interpolation points. Triangles: interpolation points where BCs are considered.

The unknown field $u$ is approximated by $\operatorname{RBF}$ interpolation $\left(\phi_{j}(\mathbf{x}):=\right.$ $\left.\phi\left(\left\|\mathbf{x}-\mathbf{x}_{\mathbf{j}}\right\|\right)\right)$

$$
u(\mathbf{x})=\sum_{j=1}^{N} \alpha_{j} \phi_{j}(\mathbf{x})
$$


where $N=J_{i n}+J_{b n}$, with $J_{b n}$ nodes at the neighbouring sub-domains of a given integration sub-region forming the interpolation stencil (where the interpolation stencil at each sub-domain is constructed by the integration sub-region and its neighbouring sub-domains as shown in Fig. 2). and $J_{\text {in }}$ nodes inside the integration sub-domain considered in the stencil. For these local interpolations various integration types of stencil configurations are defined (see Fig. 2).

The approach presented in this work can be considered as Regular since the integral equations are only applied to internal points, and Local since the unknown field $\mathrm{u}$ is interpolated locally. To convert the domain integral in Eq. (7) into a boundary integral the Dual Reciprocity Method is considered. Therefore, the approach presented in this work will be referred as Regular Local Dual Reciprocity Method (RL-DRM). Besides, the proposed approach is a type of meshless scheme since no matching conditions is directly imposed at the sub-domain interfaces, which requires a connectivity algorithm for its implementation. In the present case, as well as in the Popov and Bui RBIEM approach [8], these matching conditions are imposed at the local interpolation by considering the corresponding extension of the interpolation functions across the integration sub-domains defining the interpolation stencil.

Consequently, no matching conditions over common sub-domain interfaces are necessary in this approach, substantial simplifying the numerical implementation of this type of approach. It is important to mention that in the proposed approach the boundary conditions of the problem are directly imposed in the local interpolation stencils instead of at the integration surface as in classical BEM, additionally simplifying the numerical implementation of the proposed approach.

Depending on the relative position of the stencil with respect to the domain different situations occur. When a stencil is internal, as in stencil 1 in Fig. 2, no interpolation points are considered on the stencil boundaries (crosses and empty circles in stencil 1, Fig. 2). On the other side, when a stencil is on the boundary of the whole domain points on the boundary of the stencil are considered for the interpolation (filled triangular symbols on the domain boundaries plus empty circles and crosses, in stencils 2, 3 and 4 in Fig. 2). Regarding the boundary conditions $(\mathrm{BC})$ of the problem different types of interpolation are applied. When the boundary points of the stencil belong to the Dirichlet type BC a simple interpolation is considered. When any of the boundary points of the stencil is of a Neumann type of BC a symmetric interpolation (Hermit) is used at such nodal point, considering the normal derivative of the RBF in that boundary point.

If $u=\left(u_{1}, u_{2}, \ldots, u_{N}\right)$ it is possible to express $\alpha$ from $A$ and $u, \alpha=\left[A^{-1}\right][u]$, so:

$$
u(\mathbf{x})=\left[\phi_{j}(\mathbf{x})\right]\left[A^{-1}\right][u]
$$

For the DRM interpolation it is considered:

$$
b(\mathbf{x})=\sum_{j=1}^{N} \beta_{j} \varphi_{j}(\mathbf{x})
$$


in this work $\left\{x_{j} \in X, j=1,2, \ldots, J_{b n}+J_{i n}\right\}$, similarly to equation (21) beta is written as: $\beta=\left[\widetilde{A}^{-1}\right][b]$, where vector $[b]$ written as:

$$
[b]=\left(\left[-V_{i} \frac{\partial \varphi_{j}}{\partial x_{i}}\right]+\left[-k \varphi_{j}\right]\right)\left[A^{-1}\right][u]
$$

then reaching

$$
\beta=\left[\widetilde{A}^{-1}\right]\left(\left[-V_{i} \frac{\partial \varphi_{j}}{\partial x_{i}}\right]+\left[-k \varphi_{j}\right]\right)\left[A^{-1}\right][u]
$$

Using (14) and (16) in the integral formulation (7) with $\xi=x_{i}$ center of $\Omega_{i}$ the discretized for the unknown $u_{i}=u\left(\mathbf{x}_{\mathbf{i}}\right)$ is given as

$$
u_{i}=\sum_{j=1}^{N} \alpha_{j} H_{i j}-\sum_{j=1}^{N} \alpha_{j} G_{i j}-\sum_{j=1}^{N} \beta_{j}\left\{\widetilde{\varphi}_{j}\left(\mathbf{x}_{\mathbf{i}}\right)-\widetilde{H}_{i j}+\widetilde{G}_{i j}\right\}
$$

In matrix notation:

$$
u=H[\alpha]-G[\alpha]-F[\beta]
$$

where $H_{j i}=\int_{\Gamma_{i}} q^{*}\left(\mathbf{x}, \mathbf{x}_{\mathbf{i}}\right) \phi_{j}(\mathbf{x}) d \Gamma_{x}, G_{j i}=\int_{\Gamma_{i}} u^{*}\left(\mathbf{x}, \mathbf{x}_{\mathbf{i}}\right) \frac{\partial \phi_{j}}{\partial n}(\mathbf{x}) d \Gamma_{x}$ and $F_{j i}=\widetilde{\varphi}_{j}\left(\mathbf{x}_{\mathbf{i}}\right)-\int_{\Gamma_{i}} q^{*}\left(\mathbf{x}, \mathbf{x}_{\mathbf{i}}\right) \widetilde{\varphi}_{j}(\mathbf{x})-u^{*}\left(\mathbf{x}, \mathbf{x}_{\mathbf{i}}\right) \frac{\partial \widetilde{\varphi}_{j}}{\partial n}(\mathbf{x}) d \Gamma_{x}$. Using (15) and (18) is obtained:

$$
u=\left(H\left[A^{-1}\right]-G\left[A^{-1}\right]-F\left[\widetilde{A}^{-1}\right]\left(\left[-V_{i} \frac{\partial \varphi_{j}}{\partial x_{i}}\right]+\left[-k \varphi_{j}\right]\right)\left[A^{-1}\right]\right)[u]
$$

All the integrals in the above formulations are regulars, since the collocation points are located inside the integration sub-domains, and they are evaluated through the Gauss-Legendre quadrature.

\section{Numerical results}

In this section we present the numerical results for a boundary value problem solved with the RL-DRM method. The governing equation for the case we are presenting in this paper is a one-dimensional convection-diffusion problem with reaction term and a variable velocity field parallel to the $\mathrm{x} 1$-axis

$$
D \frac{\partial^{2} u(\mathbf{x})}{\partial x_{i}^{2}}+V_{1} \frac{\partial u(\mathbf{x})}{\partial x_{1}}+k u(\mathbf{x})=0
$$

satisfying the following boundary conditions

$$
(B C s)\left\{\begin{array}{cc}
u\left(0, x_{2}\right)=U_{0} & u\left(1, x_{2}\right)=U_{1} \\
\frac{\partial u}{\partial x_{2}} \mid x_{2}=-0.1 & \frac{\partial u}{\partial x_{2}} \mid x_{2}=-0.1
\end{array}\right.
$$


The convective velocity field is

$$
V_{x_{1}}=\ln \frac{U_{1}}{U_{0}}+k\left(x_{1}-\frac{1}{2}\right) ; \quad V_{x_{2}}=0
$$

corresponding to the flow of a hypothetical compressible fluid with a density variation inversely proportional to the velocity field. The analytical solution of the above boundary valued problem for $D=1 \mathrm{~m} 2 / \mathrm{s}$ is given by

$$
u(\mathbf{x})=U_{0} \exp \left\{\frac{k}{2} x_{1}^{2}+\left(\ln \frac{U_{1}}{U_{0}}-\frac{k}{2}\right) x_{1}\right\}
$$

For the numerical analysis this one-dimensional problem will be considered as two-dimensional in a rectangular domain $Q=[0,1] \times[-0.1,0.1]$

To evaluate the performance of the RL-DRM we present numerical results for different values of the decay parameter $k=40,100$ and 200. For $k=40$ the RL-DRM solutions are analyzed by comparing the numerical results with the analytical solution (25) and with numerical results obtained by Popov and Bui [8] applying the RBIEM method. Figure 3, for the case of $k=40$, shows very good agreement with the analytical solution for the potential field and its derivative, respectively. It can be observed that numerical results for RL-DRM reaches good solutions on the boundaries of the domain, where numerical results are reported to be inaccurate with RBIEM [8].

Table 1 shows the L2-norm error for $U$ and dU obtained with RL-DRM for different discretizations of the domain, results obtained with RBIEM [8] are also included in table for comparison. Since RL-DRM yields 1 integral equation per node and RBIEM gives 3 equations per node, the number of equations (NE) is included in Table 1 to clarify the comparison between the two methods.

Results for RL-DRM with $80 \mathrm{NE}$ (80x1) with 80 sub-domains and only one evaluation point per sub-domain are shown in Table 1 to emphasize the improvement of the approximated solution while increasing the internal points
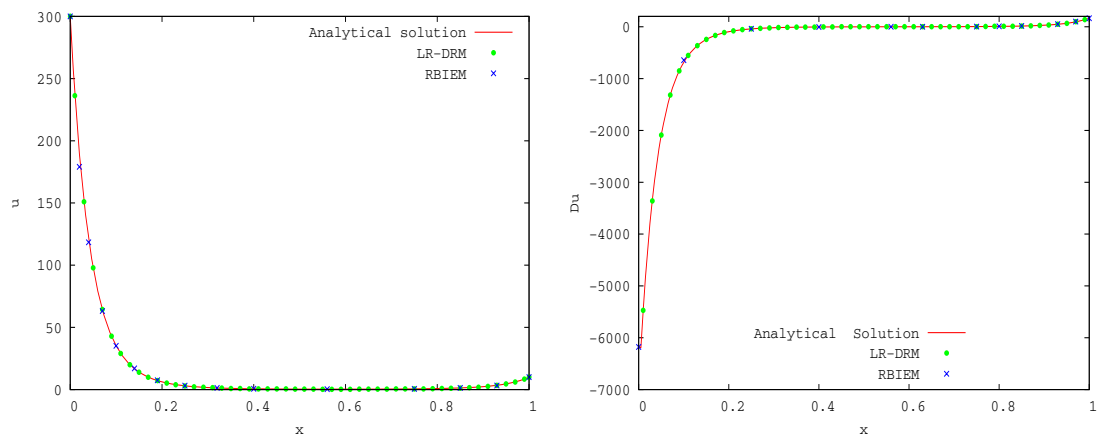

Figure 3: Left) U profiles. Right) dU profiles. Full line: analytical solution. Circles: RL-DRM.Crosses: RBIEM. $k=40$. 
Table 1: RL-DRM: L2-norm error of U and dU. RBIEM: L2-norm error of U. NN: number of nodes, NE: number of equations, nsd: number of sub-domains, nip: number of internal points per sub-domain. $k=40$.

\begin{tabular}{|c|c|c|c|c|}
\hline Method & NN (nsdxnip) & NE & L2 error U(\%) & L2 error dU(\%) \\
\hline RL-DRM & $80(80 \times 1)$ & 80 & 17.63 & 29.92 \\
& $360(40 \times 5+160 \times 1)$ & 360 & 1.48 & 15.40 \\
& $400(80 \times 5)$ & 400 & 0.85 & 1.25 \\
& $440(60 \times 5+140 \times 1)$ & 440 & 0.57 & 2.44 \\
\hline RBIEM & 95 & 285 & 5.35 & not reported \\
& 185 & 555 & 4.73 & not reported \\
& 689 & 2067 & 1.48 & not reported \\
& 1513 & 4539 & 0.75 & not reported \\
& 2657 & 7971 & 0.48 & not reported \\
\hline
\end{tabular}

per sub-domain, as is the case for $400 \mathrm{NE}$ (80x5 Fig. 1), corresponding to 80 sub-domains but 5 evaluation points per sub-domain, reducing the error for $\mathrm{U}$ from $17.63 \%$ to $1.48 \%$, respectively. Solutions for 360 and $440 \mathrm{NE}$ correspond to p-adaptive strategies, considering nodal enrichment where the absolute errors of previous uniform discretizations are higher. It must be mentioned that the small lost of accuracy of the L2-norm error for $440 \mathrm{NE}$ for the $\mathrm{dU}(2.44 \%)$ is due to the proximity of the nodes which implies ill-conditioning of the local system of equations. This problem could be overcome either increasing the numerical precision or improving the linear solver.

It is fair to mention that similar improvement on the solution can be achieved with the RBIEM scheme by using more than one integration point for sub-domain instead of only one as original proposed in [9].

Comparing the two methods in Table 1, it can be observed that RL-DRM reaches a $0.57 \mathrm{~L} 2$-error for $440 \mathrm{NE}$, while RBIEM attains a $0.48 \mathrm{~L} 2$-error for $7971 \mathrm{NE}$.

Figure 4 shows the computed RL-DRM solutions for the potential field U, with $k=100$ and $k=200$, which are in very good agreement with the analytical solution.

\section{Conclusions}

A method based on the DRM approach considering only regular integrals and local RBF interpolation has been presented for the solution of the convectiondiffusion equation. Numerical solutions for different Peclet numbers are shown to be in very good agreement with analytical solutions. Even when in this work the discretization of the domain is based on rectangular sub-domains, the RL-DRM 

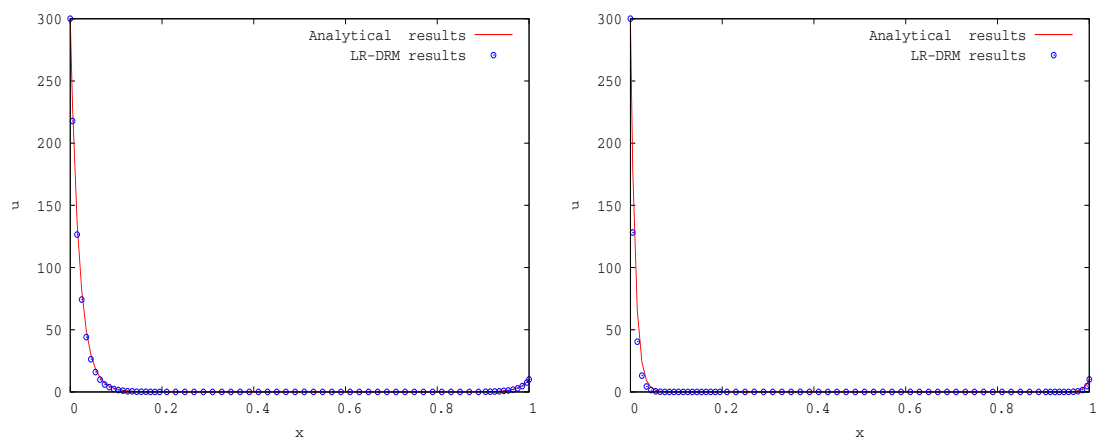

Figure 4: U profiles. Full lines: analytical solution. Circles: RL-DRM. Left) $k=$ 100. Right) $k=200$.

is not a sub-domain technique as no matching conditions are necessary. The RLDRM can easily add internal points per sub-domain in an adaptive fashion, highly increasing the accuracy of the method. This new technique can be considered as meshless since there is no restriction on the choice of the nodes where the integral equations are applied and no a direct implementation of the sub-domain matching conditions are required.

\section{References}

[1] Nardini, D. \& Brebbia, C.A., Boundary element integral formulation of mass matrices for dynamic analysis (Chapter 7). Topics in Boundary Elements Research, ed. C.A. Brebbia, Springer-Verlag: Berlin and New York, pp. 191207, 1985.

[2] Popov, V. \& Power, H., A domain decomposition in the dual reciprocity approach. Boundary Element Communications, 7/1, pp. 1-5, 1996.

[3] Kansa, E.J. \& Carlson, R.E., Radial basis functions: a class of grid-free, scattered data approximations. Computational Fluid Dynamics, 3/4, pp. 479496, 1995.

[4] Florez, W., Power, H. \& Chejne, F., Multi-domain dual reciprocitybemapproach for thenavier-stokes system of equations. Communications in Numerical Methods in Engineering, 16, pp. 671-681, 2000.

[5] Portapila, M. \& Power, H., A convergence analysis of the performance of the drm-md boundary integral approach. International Journal for Numerical Methods in Engineering, 71, pp. 47-65, 2007.

[6] Wright, G.B. \& Fornberg, B., Scattered node compact finite difference-type formulas generated from radial basis functions. Journal of Computational Physics, 212, pp. 99-123, 2006. 
[7] Orsini, P., Power, H. \& Morvan, H., Improving volume element methods by meshless radial basis function techniques. Computer Modeling in Engineering and Sciences, 23(3), pp. 187-207, 2008.

[8] Popov, V. \& Bui, T.T., A meshless solution to two-dimensional convectiondiffusion problems. Engineering Analysis with Boundary Elements, 34, pp. 680-689, 2010.

[9] Bui, T.T. \& Popov, V., Domain decomposition boundary element method with overlapping sub-domains. Engineering Analysis with Boundary Elements, 33, pp. 456-466, 2009. 